\title{
Aprendizaje acelerado y desarrollador: alternativa para perfeccionar la expresión oral de la lengua inglesa
}

\author{
Gonzalo Rafael Almanza Casola y Arely Beatriz Ascuy Morales \\ Universidad de Ciego de Ávila, Cuba \\ Javier Villoria Prieto y Jesús Muros Navarro \\ Universidad de Granada
}

Recibido: 9 diciembre 2007 / Aceptado: 10 febrero 2008

ISSN: $1697-7467$

\begin{abstract}
RESUMEN: En este artículo se presenta uno de los más importantes resultados de una investigación desarrollada en la Universidad de Ciego de Ávila, Cuba, cuya finalidad era perfeccionar el proceso de enseñanza aprendizaje de la lengua inglesa en los estudiantes de primer año de las carreras de Contabilidad y Finanzas, Informática y de Turismo. Partimos de las insuficiencias que presenta este proceso en la referida universidad que dificultan el establecimiento de una comunicación eficiente en este idioma; para su solución se elaboró una Alternativa Metodológica dirigida a perfeccionar la expresión oral en la lengua inglesa, fundamentada en las concepciones actuales del aprendizaje acelerado y en las aportaciones del paradigma histórico cultural acerca del aprendizaje desarrollador que, unidos a diferentes métodos y técnicas participativas, garantizan el cumplimiento de los objetivos propuestos. Esta investigación responde a los postulados de la educación permanente presentada por la UNESCO en el informe «Aprender a Ser». Desde el punto de vista del método científico, la forma en que se realizó la investigación y se recogieron los datos es afín al diseño preexperimental de un grupo con pretest y postest. Para llevar a cabo las indagaciones empíricas y recoger los datos se emplearon diferentes técnicas e instrumentos de investigación, en correspondencia con los indicadores que se establecieron para medir el aprendizaje acelerado y desarrollador del idioma inglés. Entre los principales resultados alcanzados se encuentran la influencia positiva que se logró en la formación integral de los estudiantes y el aumento de la competencia expresiva oral junto al resto de las demás habilidades del idioma.
\end{abstract}

Palabras clave: aprendizaje desarrollardor, sujestopedia, aprendizaje acelerado, expresión oral y lengua inglesa.

ABSTRACT: This article presents one of the most important outcomes of a research
carried out in the University Of Ciego de Ávila Cuba, with the purpose of improving the
English language teaching-learning process in first-academic-year-students of Accounting
and Finances, Informatics and Tourism courses. We started from the inadequacies that
this process presents in this university which hinder the establishment of a steady
communication in that language. In order to solve such problem, a Methodological Alternative
was elaborated directed to improve the oral expression in the English language, with its
foundations laid on the present day conceptions of the accelerated learning as well as on 
the contributions of the historical-cultural paradigm about the developing learning, which together with different methods and participative techniques; guarantee the accomplishment of the goals that have been set. The research carried out responds to the permanent education's postulates presented by UNESCO in the report "Learning to Be". From the scientific method point of view, the way used to do the research and to gather data is akin to the pre-experimental design of a group with pre-test and post-test. To carry out the empirical inquiries and to gather the data, several techniques and researching tools were employed according to the indicators established to measure the accelerated learning and to develop English language. The most outstanding results include the achievement of a positive influence on the students' integral formation and the development of the oral expression as well as of the rest of the language's skills.

Key words: developing learning; suggestopaedia; accelerated learning; oral communication; English language.

\section{INTRODUCCIÓN}

El perfeccionamiento de la formación de profesionales universitarios constituye una necesidad impostergable. El egresado universitario debe ser por excelencia un transformador de la sociedad y de su entorno natural, ha de tener un pleno dominio de los contenidos de su ciencia, para poder expresar sus ideas y actuar eficaz y eficientemente en la solución de los problemas de su profesión. Una de las vías que le va a permitir acceder a la información necesaria y al uso de la tecnología es precisamente el dominio de la lengua inglesa, tanto de forma escrita como oral.

Según resultados de las investigaciones realizadas por el Departamento de Idiomas del Instituto Superior Pedagógico Manuel Ascunce Doménech (1995) y por el Departamento de Idiomas de la Universidad de Ciego de Ávila (2000), y a partir del diagnóstico que realizan los profesores del departamento de idiomas de la Universidad de Ciego de Ávila, Cuba, se puede señalar que, a pesar de que los alumnos han transitado por diferentes niveles de enseñanzas donde estudian inglés, aún presentan dificultades en el uso adecuado de las diferentes estructuras morfosintácticas y lexicales que intervienen en las funciones comunicativas estudiadas, lo que imposibilita la comunicación en la lengua extranjera.

Estas dificultades generan que el estudiante que ingresa a la universidad no pueda comprender con claridad los componentes básicos de la lengua y sea preciso un perfeccionamiento del proceso de enseñanza aprendizaje del idioma inglés de forma tal que propicie la formación y desarrollo integral de la personalidad del profesional que egresa de la universidad, lo que nos condujo al planteamiento del siguiente problema científico: ¿Cómo perfeccionar la expresión oral en la lengua inglesa de los estudiantes de las carreras de Contabilidad y Finanzas, Informática y de Turismo de la Universidad de Ciego de Ávila?

Para lograr este perfeccionamiento se propone como objetivo elaborar una alternativa metodológica para el aprendizaje acelerado y desarrollador de la lengua inglesa que propicie un perfeccionamiento de la expresión oral de los estudiantes de la UNICA.

La concepción de investigación que sustenta este trabajo se basa en los postulados de la educación permanente, presentada por la UNESCO en el informe "Aprender a ser» y se fundamenta filosófica, psicológica y pedagógicamente en las mejores tradiciones del pensamiento humanista (Castellanos, 1998). 
Esta perspectiva presupone que el investigador, que es el propio docente, pertrechado con el método científico como estrategia orientada a la producción de conocimientos, transforme su realidad, parta de la exploración y problematización del contexto educativo y construya activamente el objeto de estudio, apoyándose en referentes prácticos y teórico conceptuales.

Para llevar a cabo las indagaciones empíricas y recoger los datos necesarios se emplearon diferentes técnicas e instrumentos de investigación en correspondencia con los indicadores que se establecen para medir el aprendizaje desarrollador de los estudiantes según los criterios de Castellanos y otros (2001). Estas técnicas e instrumentos son:

- La observación como el registro sistemático, válido y confiable de comportamiento o conductas manifiestas (Hernández, 1998) fue utilizado para valorar cómo se desarrolla la actividad docente con la aplicación de la alternativa metodológica propuesta.

- La encuesta con el objetivo de conocer las opiniones e intereses de los estudiantes acerca del proceso de enseñanza del inglés y sus preferencias con respecto a la clase de idiomas y a las habilidades que en ella se forman y desarrollan, algunas reflexiones metacognitivas, relaciones significativas entre los contenidos, vinculación entre los contenidos y la vida, así como para conocer la opinión con respecto a la metodología utilizada por el profesor.

- Para la evaluación del dominio de la habilidad de expresión oral en los estudiantes se utilizaron las pruebas de comprobación del rendimiento escolar en tres plazos durante el curso escolar. A principios del curso se pasaron las pruebas para tener un diagnóstico del nivel de entrada de los estudiantes por lo que se trazó como objetivo determinar el nivel alcanzado por éstos en la expresión oral en idioma inglés durante los estudios realizados en la enseñanza Media Superior.

Se aplicó otro test al finalizar el primer semestre del curso con un carácter parcial y con el objetivo de comprobar el desarrollo en la expresión oral alcanzada por los estudiantes en el idioma inglés. Al finalizar el curso escolar los estudiantes realizaron un último test cuyo objetivo era similar al anterior.

Para la calificación de este examen se utilizó un tribunal que estuvo formado por tres profesores, además del que imparte clases en el grupo; para la evaluación por parte de este tribunal se utilizó una escala valorativa analítico-sintética que responde a una escala tipo Likert.

Se utilizó, además, un registro con las opiniones de los estudiantes durante el desarrollo del proceso educativo. Esta técnica fue tomada de la experiencia llevada a cabo por el Centro Nacional de Educación Sexual con su programa «Mi Proyecto de Vida». Consiste en llevar un registro de las experiencias de los estudiantes durante el proceso educativo, en este registro ellos expresan sus sentimientos con respecto al proceso de una forma anónima y sin la presión del profesor, es decir, espontáneamente, lo que permitió al investigador conocer qué criterios y qué vinculo emocional, así como qué valoraciones fueron asumiendo los estudiantes durante el proceso de enseñanza aprendizaje utilizando la alternativa metodológica propuesta.

Otra técnica que permitió valorar las bondades de la alternativa metodológica, así como el conocimiento y la reflexión metacognitiva de los estudiantes fue la composición. 
Los resultados obtenidos en estas dos últimas técnicas fueron procesadas a través del método cualitativo de análisis de contenido o análisis cualitativo de datos, como lo denominan Rodríguez y otros (2002).

\section{Desarrollo}

Es evidente que la enseñanza pasivo-reproductiva que estimula la escuela bajo las concepciones tradicionalistas no va a propiciar el cambio que se necesita, por lo que han surgido en el pensamiento pedagógico contemporáneo distintas alternativas que de un modo u otro han intentado solucionar los problemas que se enfrenta y que tienen como denominador común el hecho de trasladar el centro del proceso al aprendizaje del estudiante, por lo que es indispensable que éste aprenda cómo se aprende. Algunos de los autores que se han dedicado a desarrollar diferentes alternativas en la docencia caracterizadas por el empleo de variados métodos activos son Canfux y González, et.al (1995); otro ejemplo constituye el aprendizaje significativo, cuyos principales exponentes son Ausbel, Joseph, y Hanesian (1991).

En la enseñanza de lenguas extranjeras también han surgido diferentes alternativas, entre las que se encuentran las relacionadas con el aprendizaje acelerado. Esta concepción surge a partir de la puesta en práctica del método sugestopédico y, como se ha dicho anteriormente, han sido varios las propuestas metodológicas que se han realizado fundamentadas en los principios del mismo. Sobre ello se hace una valoración crítica en la tesis doctoral Aprendizaje Acelerado: Propuesta de una alternativa metodológica para el perfeccionamiento de la expresión oral de la lengua inglesa (2006).

Lozanov (1978) señala que la Sugestopedia debe entenderse como la instrucción-educación fundamentada en las leyes y los principios de la sugestión. Lo anteriormente señalado por Lozanov es argumentado por Larsen-Freeman (2001), quien plantea que el objetivo de la puesta en práctica del método sugestopédico consistía en ayudar a los estudiantes a eliminar el sentimiento de inseguridad de la supuesta incapacidad para enfrentar con éxito el aprendizaje y también para evitar una actitud negativa hacia el estudio, porque a través del mismo se pueden romper esas barreras psicológicas que entorpecen el aprendizaje.

En un inicio Lozanov (1978) justificaba la comprensión del concepto de sugestión y su aplicación en el proceso de enseñanza-aprendizaje. Sin embargo, este interés se fue perdiendo a partir de la introducción de su tercera versión del método, elaborado por él y por su ayudante Gateva en 1989. En una carta dirigida a la Alianza Internacional para el Aprendizaje (1999) plantea:

Los enfoques (ej. la relajación guiada, la imagen, los ejercicios de respiración), que son métodos tradicionales para inducir el estado hipnótico, son una forma de condicionamiento que subordinan la voluntad, limita la creatividad de los estudiantes, automatizando su pensamiento y conducta. (Lozanov, 1999: 2).

Es a partir de este momento cuando comienza a denominar su método por el nombre de Desugestopedia ${ }^{1}$.

1. En la tesis doctoral: Aprendizaje Acelerado: Propuesta de una alternativa metodológica para el perfeccionamiento de la expresión oral de la lengua inglesa (2006) se realiza una valoración crítica sobre los métodos con enfoques psicológicos que se agrupan y fundamentan en el aprendizaje acelerado. 
Entre el aprendizaje acelerado y el aprendizaje desarrollador hay varios puntos de contacto, porque responden a la escuela Histórico Cultural, aunque esto no se declara en el primero. Ambos tienen en cuenta el estado emocional del alumno, el contexto físico donde se produce el proceso de enseñanza-aprendizaje, la motivación del estudiante, la individualidad y diversidad de los sujetos y el papel del grupo en el aprendizaje (Almanza, 2006), aspectos estos asumidos por la alternativa metodológica a la que se ha hecho referencia en este artículo.

El proceso de enseñanza aprendizaje de un idioma no escapa a estas nuevas concepciones, cada día se hace más evidente la necesidad de dominar un idioma extranjero para propiciar un desarrollo más eficiente y eficaz del profesional en formación.

Aunque en la actualidad el discurso teórico hace referencia a la necesidad de motivar y cultivar el interés de los estudiantes, en la práctica pedagógica con frecuencia sólo se crean vínculos formales a partir del rígido carácter normativo que generalmente se fomenta en la escuela.

En el proceso de enseñanza-aprendizaje de un idioma extranjero también están presentes esos problemas, de ahí que a pesar de que en la enseñanza media y media-superior el estudiante recibe un total de 660 horas clases, el resultado no suele ser alentador e ingresa en la universidad con una serie de limitaciones motivadas por serias dificultades en el dominio de las estructuras morfosintácticas y lexicales que afectan notablemente la comunicación en la lengua extranjera. Problemas que, a pesar del perfeccionamiento constante del proceso de enseñanza-aprendizaje que exige la sistemática validación de los programas de las asignaturas en la enseñanza superior, no se resuelven; los estudiantes continúan con limitaciones severas en el dominio del idioma y, una vez egresados, les resulta engorroso establecer una comunicación estable sobre un tema específico, incluyendo los de su especialidad, lo que evidencia que los cambios curriculares constituyen condiciones necesarias, pero no suficientes para mejorar la calidad de la educación.

Ante esta situación se considera necesario un perfeccionamiento del proceso, no desde el discurso sino desde la práctica pedagógica, y esto exige un cambio en las concepciones de los profesores que enseñan idioma.

Es por ello por lo que se opta por una alternativa metodológica que se conceptualiza como una opción que asumen el profesor, los estudiantes y el grupo en el proceso de enseñanza-aprendizaje de un idioma (inglés) para fines específicos que tiene como centro la interacción de éstos, donde la motivación y la actuación conscientes son elementos muy relevantes (Almanza, 2001), pues dan al traste con las dimensiones del aprendizaje desarrollador ofrecida por Castellanos et al. (2001): la activación-regulación, la significatividad y la motivación para aprender.

Cuando se analiza el proceso de enseñanza-aprendizaje hay que tener en cuenta sus dos polos: el aprendizaje como la actividad que ejecuta el estudiante que se está formando y la enseñanza, actividad que ejecuta el profesor, quien es responsable de orientar y guiar ese aprendizaje. Ambos actúan sobre una materia de estudio (Álvarez, 1992) como un proceso único, donde constantemente se interiorizan y exteriorizan los aprendizajes de cada uno de los sujetos que intervienen y del grupo con su dinámica de actuación, donde profesor y estudiantes enseñan y aprenden al mismo tiempo.

El aprendizaje desarrollador, término que se deriva de la categoría vigotskiana «Enseñanza Desarrolladora», garantiza que el individuo se apropie activa y creadoramente de la 
cultura, propiciando el desarrollo de un autoperfeccionamiento constante de su autonomía y autodeterminación, en íntima conexión con los procesos de socialización, compromiso y responsabilidad social y, según la opinión de Castellanos (2001), debe cumplir tres criterios básicos:

1. Promover el desarrollo integral de la personalidad del educando, lo que quiere decir que no sólo debe activar la apropiación de conocimiento, destrezas y capacidades intelectuales, sino también la formación de sentimientos, cualidades, valores, convicciones e ideales.

2. Garantizar el tránsito progresivo de la dependencia a la independencia y de ésta a la autorregulación, desarrollar en el sujeto la capacidad de conocer, controlar y transformar creadoramente su propia persona y el medio.

3. Desarrollar la capacidad para realizar aprendizajes a lo largo de toda la vida a partir del dominio de habilidades y estrategias para aprender a aprender y de la necesidad de una autoeducación constante.

Partiendo de estos criterios, esta alternativa metodológica propone como finalidad lograr un aprendizaje desarrollador en los estudiantes utilizando los aportes metodológicos del aprendizaje acelerado de lenguas.

Todo esto impone cambios importantes en la concepción del proceso de enseñanzaaprendizaje en sentido general, teniendo en cuenta los criterios de Parra (2000). Se propone entonces que el programa docente, más que un rígido documento que traza las pautas a seguir en el proceso enseñanza-aprendizaje, se convierta en un proyecto aceptado por el profesor y los estudiantes, un proyecto sujeto a cambios que estarán derivados de las necesidades del aprendizaje profesional, y que debe poder ajustarse a la marcha del proceso, convirtiendo al profesor en un orientador que mueva al estudiante a niveles superiores de desarrollo.

Los objetivos han de formularse en función del estudiante, es decir, en términos del aprendizaje. Siguiendo la posición de Mitjáns (1995), hay que lograr que los objetivos favorezcan en la mayor medida posible la implicación de los alumnos, para lo cual es necesario lograr su participación en la determinación y formulación de los mismos, propiciando la reflexión individual y grupal. Esto permite conocer qué visión tiene el estudiante de la asignatura y cuáles son sus expectativas, tanto de forma individual como grupal; además es una de las formas de incidir sobre los procesos de reflexión y regulación metacognitiva, pues el hecho de que el estudiante tenga conciencia de sus metas de aprendizaje, se las haya propuesto sobre la base de la reflexión acerca de sus posibilidades de lograrlas y los esfuerzos y estrategias a desplegar para ello, influyen positivamente en su desarrollo integral.

Los métodos de enseñanza-aprendizaje no existen sin los objetivos ni conocimientos sobre los que deben sustentarse, el objetivo sólo puede alcanzarse por medio de un método y sobre la base de un conocimiento (Bermúdez y Rodríguez, 1996). De ahí que si se utiliza un mecanismo para la determinación de los objetivos donde se logre una implicación de los estudiantes en el proceso, los métodos no pueden responder a una enseñanza tradicional. Se requiere entonces la utilización de métodos activos o productivos de enseñanza.

La fundamentación descrita justifica que en esta alternativa se proponga la utilización de las concepciones metodológicas del aprendizaje acelerado, que se desprende del método sugestopédico pero lo supera a partir de su contextualización y enriquecimiento con los 
resultados investigativos y la experiencia docente. Se tienen en cuenta, además, las características de los sujetos que intervienen en él de forma interactiva y se usan diferentes métodos tales como los juegos de roles, la simulaciones, el método de discusión en todas sus variantes (discusión plenaria, discusión en pequeños grupos, discusión conferencia, discusión confrontación, mesa redonda, discusión panel) (CPES, 1995) y técnicas para el desarrollo de habilidades, como son: los mensajes escritos del amigo secreto, chistes, adivinanzas, trabalenguas, entre otros.

El uso de tales técnicas contribuye a la elevación de la calidad del proceso comunicativo, provocando importantes cambios en la actitud de los alumnos con respecto a la apropiación de los conocimientos, favoreciendo una mayor implicación personal y mayor desarrollo de la motivación; se logra de esta forma, siguiendo algunos de los presupuestos de la Sugestopedia, desinhibir al estudiante de las tensiones que representa el aprendizaje de una lengua extranjera, mostrándole que puede ser un proceso agradable y fácil.

Otro punto importante que exige cambio es la evaluación, de forma tal que en el proceso los estudiantes asuman conscientemente sus potencialidades, logros y posibilidades, para lo cual resulta importante adoptar de forma conjunta los criterios e indicadores de evaluación que deberán regir, así como el establecimiento del compromiso personal del estudiante.

Es imprescindible organizar el proceso de modo que los estudiantes puedan interactuar entre sí atendiendo a su nivel de desarrollo actual y potencial, lo que exige la resolución de tareas que deben caracterizarse por propender a exigencias mayores; por último, se debe alcanzar el compromiso del alumno con el proceso como sujeto activo de éste. Todo ello permite afirmar que la alternativa propuesta se caracteriza por:

- Partir de las necesidades básicas de aprendizaje de los educandos, teniendo en cuenta sus características individuales, su desarrollo actual y potencial y las condiciones en las que vive y se desarrolla.

- Centrarse en el alumnado promoviendo la construcción personalizada de los conocimientos.

- El predominio de la comunicación y el intercambio de saberes.

- Destacar el papel del grupo en el aprendizaje, garantizando un clima emocional favorable en el aula.

- Concebir a los estudiantes como sujetos activos del proceso que participan en la construcción de sus saberes.

- Romper esquemas y hábitos de trabajo directivos, autoritarios por parte del profesor, para asumir la función de estimular la tarea grupal e individual, brindar a los estudiantes la posibilidad de la libre elección de la actividad de estudio, dándoles variantes para ésta, así como un lugar en el colectivo. El docente estimula sus posibilidades creativas, descubre y anima sus potencialidades, desarrolla la independencia, eleva la responsabilidad por el producto de su trabajo, por sus acciones y conductas.

Todo esto se concreta en los pasos metodológicos que a continuación se exponen, con respecto a los cuales es importante dejar claro que solo se presentan las principales recomendaciones de la alternativa metodológica. 


\section{Primera clase del curso}

- Aula al inicio de la actividad. Se deben colocar informaciones alrededor del aula relacionadas con el nuevo contenido para que se ponga de manifiesto el aprendizaje periférico. Los asientos de los estudiantes deben estar en posición de $\mathrm{U}, \mathrm{V}$ o círculo para que se facilite la comunicación.

- Aplicar técnicas de animación para crear un ambiente fraterno y de confianza. Se sugieren: «La historia loca», «Mar adentro y mar afuera» y «El alambre pelado», entre otras.

- Concentración y presentación: con un fondo musical que invite a la relajación. Se realiza una de las siguientes técnicas: presentación en parejas, bailes de presentación, la tela araña u otras con iguales objetivos.

Posteriormente se realiza un ejercicio encaminado a conocer qué visión tienen los estudiantes de la asignatura y cuáles son sus expectativas tanto de forma individual como grupal. Finalizado esto, se lleva a cabo la negociación de la evaluación, que consiste en analizar con los estudiantes cómo se realizará la misma.

- Por último se aplica un test diagnóstico para determinar el desarrollo alcanzado por los estudiantes en niveles de enseñanzas precedentes.

\section{Segunda clase del curso}

- Una vez que se salude a los estudiantes, se sugiere un ejercicio de animación e inmediatamente el profesor aplica una actividad de comprobación de conocimiento sencilla para que sea resuelta con relativa facilidad por el estudiantado, esto tiene como objetivo ofrecerles un sentido de seguridad y confianza.

- Se propone trabajar con la nueva identidad y la correspondiente profesión que deben asumir los estudiantes durante el curso. Se pueden emplear diferentes técnicas para introducir el nuevo contenido.

- Se indica que al final se les proponga a los alumnos una fiesta simulada donde nadie se conoce y así ejercitar el nuevo material impartido.

\section{Tercera clase del curso y primera de cada unidad nueva}

- Introducción (Introduction): se realiza un warm up, revisión de actividades previas entre otras.

- Desarrollo (Development): se sugieren ejercicios basados en clases anteriores para practicar y consolidar los conocimientos previos alcanzados. Para introducir el nuevo contenido se sugiere:

- Primera audición (First Listening). Esta es de familiarización y sin apoyo visual. Una vez realizada la misma se aplica un ejercicio de comprobación global.

- Segunda audición (Second Listening). Los estudiantes escuchan el nuevo material pero siguen con la vista el texto. El profesor puede aprovechar este momento para realizar la explicación del nuevo contenido, pero podría hacerlo, si lo prefiere, después de finalizada la primera audición.

- Tercera audición (Third Listening) y comprobación. Se finaliza la clase con la sugerencia de que el material introducido se lea antes de acostarse y recién se levanten en la mañana. 
- Las próximas clases serán de ejercitación y para ellas deben tenerse en cuenta los cuatro niveles de asimilación del conocimiento. Es importante que se mantenga en el aula un estado de regocijo y disfrute durante el desarrollo de las actividades propuestas.

El número de estas clases de ejercitación dependerá de las necesidades reales de los estudiantes.

A continuación se ofrecen de forma sintetizada algunos de los resultados obtenidos en el desarrollo de la investigación llevada a cabo y después de haberse puesto en práctica la alternativa metodológica.

El registro de experiencia de los alumnos («Historia de los participantes») permitió una retroalimentación sistemática y cualitativa del proceso de enseñanza-aprendizaje a partir de la opinión de los mismos. Se recogieron 283 opiniones durante el curso 2005-2006, todos los estudiantes participaron, aunque se debe señalar que unos fueron más activos que otros. Estos resultados fueron evaluados utilizando el método de análisis cualitativo de los datos o análisis de contenido, como se ha señalado.

Una vez realizado el análisis de las opiniones ofrecidas por los estudiantes, se pudo determinar la frecuencia con que las categorías motivación, valor afectivo, aprendizaje, comunicación, evaluación del profesor y metacognición, aparecen en el registro de experiencia.

En la siguiente tabla, se dan a conocer los resultados detectados en cada una de estas categorías:

Tabla 1: Frecuencia con que aparecen las categorías en el registro de experiencia.

\begin{tabular}{|l|c|c|}
\hline \multicolumn{1}{|c|}{ Categorías } & $\begin{array}{c}\text { Frecuencia con que aparece en el } \\
\text { registro de experiencia }\end{array}$ & $\%$ \\
\hline Motivación & 77 & $27,02 \%$ \\
\hline Valor Afectivo & 126 & $44,52 \%$ \\
\hline Aprendizaje & 108 & $42,68 \%$ \\
\hline Comunicación & 21 & $7,42 \%$ \\
\hline Evaluación del Profesor & 124 & $43,81 \%$ \\
\hline Metacognición & 9 & $3,18 \%$ \\
\hline
\end{tabular}


En la gráfica 1 se muestra el orden jerárquico de las categorías descrita en la tabla anterior.

Gráfica 1: Frecuencia con que aparecen las categorías en el registro de experiencia.

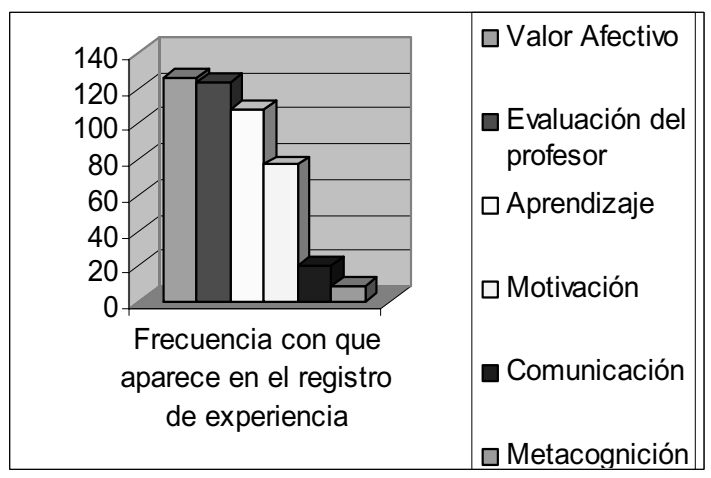

Lo más relevante en las opiniones emitidas por los estudiantes es el elevado vínculo emocional que se produce con el empleo de la alternativa, es decir, se refiere a cómo la alternativa metodológica propuesta se vincula con la satisfacción e insatisfacción de sus necesidades, expresado de forma positiva, negativa o ambivalente.

Claro está que en este caso las expresiones han estado en un $100 \%$ vinculada con valores positivos.

A los resultados de la aplicación de las pruebas de comprobación del rendimiento escolar se aplicó la prueba T. En la gráfica II se aprecia cómo las medias aritméticas ascendieron significativamente: en los resultados iniciales el valor es de 2,30 (de un máximo de 5) mientras que en los resultados finales es de 3,64. De la misma forma la desviación típica inicial es mayor que la final lo que permite inferir que las notas finales están más concentradas alrededor de la media mientras que los resultados iniciales denotan una mayor dispersión de los datos, es decir, que los niveles de desarrollo de los estudiantes estaban más dispersos, menos equilibrados.

Gráfica 2. Medias iniciales y finales de la muestra total.

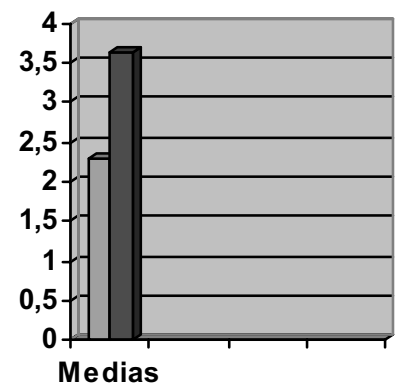


En el resultado de la prueba $\mathrm{T}$ el valor de Sig es menor que por lo que se rechaza la hipótesis nula con un 95\% de iabilidad, esto permite inferir que existen diferencias significativas entre las medias inicial y final. Como se ha podido apreciar, la media inicial es menor que la final, lo que verifica la hipótesis planteada: la implementación de una alternativa metodológica para el aprendizaje desarrollador y acelerado de la lengua inglesa para fines específicos que se propone en este trabajo posibilita el perfeccionamiento de la expresión oral de los estudiantes.

\section{Conclusiones}

Durante el desarrollo de la investigación llevada a cabo y a partir de los resultados obtenidos se llega a las siguientes conclusiones:

- Se constató que la alternativa metodológica para el aprendizaje acelerado y desarrollador de la lengua inglesa propició un perfeccionamiento de la expresión oral de los sujetos.

- Se promueve un elevado nivel de motivación entre los estudiantes, lo que se logra a partir de la creación de un ambiente agradable, dinámico y divertido en el desarrollo de proceso docente.

- Se influye positivamente en la formación integral de los estudiantes, no sólo en el desarrollo de las habilidades del idioma, lo que repercute directamente en la esfera cognitivo-instrumental de la personalidad, sino también en la esfera afectivo-valorativa y motivacional, lo que se fundamenta, además, en el desarrollo de la autovaloración de los estudiantes, en el crecimiento de su autoestima, en la pérdida de barreras psicológicas que afectaban el aprendizaje, en las relaciones interpersonales que se solidifican y cohesionan en el trabajo en grupo y en las relaciones alumno profesor.

\section{Bibliografía}

Almanza, C. G. R. (2001). Alternativa Metodológica para una Enseñanza Participativa de la Lengua Inglesa para Fines Especificos en la Facultad de Contabilidad y Finanzas. Tesis presentada para la opción del Título de Master en Educación Superior. Universidad de Ciego de Ávila, Cuba.

Almanza Casola, G. (2006). Aprendizaje Acelerado: Propuesta de una Alternativa Metodológica para el perfeccionamiento de la expresión oral de la lengua inglesa. Tesis doctoral. Granada: Universidad de Granada.

Álvarez De Zayas, C. (1992). La Escuela en la vida. La Habana: Ed. Félix Varela.

Ausubel, D., Novak, J. y Hanesian, H. (1991). Psicología Educativa. Un punto de vista cognitivo. México: Editorial Trillas. Quinta reimpresión.

Bermúdez Sarguera, R. y Rodríguez Rebustillo, M. (1996). Teoría y Teoría y Metodología del aprendizaje. Ciudad de La Habana: Ed. Pueblo y Educación.

Canfux, V. et al. (1996). Tendencias pedagógicas contemporáneas. La Habana: EPES.

Castellanos Simonds, B. (1998). Investigación Educativa. La Habana.

Castellanos Simonds, D. (2001). Hacia una concepción del aprendizaje desarrollador. La Habana. 
Castellanos Simonds, D. et al. (2001). Hacia una Concepción del Aprendizaje desarrollador. Ciudad de la Habana: Ed. Colección Progreso.

Cepes (1995). Los métodos Participativos ¿Una nueva concepción de la enseñanza? Ciudad de la Habana: Universidad de la Habana.

González, O. (S.F). La Escuela Histórico Cultura. Material mimeografiado.

Hernández Sampieri, R. (S/F). Metodología de la Investigación. Ciudad de México: Ed. McGrawHill.

Lozanov, G. (1978). Suggestology and Suggestopedy. Theory and Practice. UNESCO.

Lozanov, G. (1999). Carta dirigida a la Alianza Internacional para el Aprendizaje.

Lozanov, G. (2003). Suggestology and Outlines of Suggestopedy. London: Routledge.

Lozanov, G. y Gateva E. (1989). The Foereign Language teacher's Suggestopedic Manual. Gordon and Breanch Science Publishers. Metodología del aprendizaje. Ciudad de La Habana: Ed. Pueblo y Educación.

Mitjans Martínez, A. (1995). Creatividad, personalidad y educación. La Habana: Ed. Pueblo y Educación.

Parra Vigo, I. (2000). La profesionalización Pedagógica del Maestro en formación en un proceso de enseñanza aprendizaje desarrollador. ISP Enrique J. Varona. Ponencia. La Habana: Ed. Pueblo y Educación.

Rodríguez Gómez, G. et al. (2002). Metodología de la Investigación Cualitativa. Santiago de Cuba: Ed. PROGRAF.

Sierra Salcedo, R. (1997). Grupo Autodirigidos en Educación. Tesis presentada en opción del título de Master. Facultad de Ciencias de la Educación. Instituto Superior Pedagógico Enrique José Varona. Ciudad de la Habana. 